VOL. $9(\mid 973), 4|1-4| 5$.

\title{
Characters for summary functions associated with cartesian products
}

\section{Peter D. Finch and Elizabeth Heathcote}

\begin{abstract}
In deriving macroscopic descriptions of microscopic phenomena one often uses a vector valued summary function which is defined on a cartesian product in terms of component summary functions. We show that any character of such a sumary function is the product of characters of its component summary functions.
\end{abstract}

\section{Introduction}

We adopt without further comment the notation and terminology of Finch [1]. In many practical situations one is considering two non-empty sets $X^{\prime}$ and $X^{\prime \prime}$ together with a universal $M^{\prime}$-valued summary function $\xi^{\prime}: X_{*}^{\prime} \rightarrow M^{\prime}$ and a universal $M^{\prime \prime}$-valued summary function $\xi^{\prime \prime}: X_{*}^{\prime \prime} \rightarrow M^{\prime \prime}$. One's immediate interest is in the cartesian product $X=X^{\prime} \times X^{\prime \prime}$ and the universal $M^{\prime} \times M^{\prime \prime}$-valued summary function $\xi: X_{*} \rightarrow M^{\prime} \times M^{\prime \prime}$ defined by (1.1) $\forall n \geq 1: \xi_{n}\left(x_{1}^{\prime}, x_{1}^{\prime \prime}\right)\left(x_{2}^{\prime}, x_{2}^{\prime \prime}\right) \ldots\left(x_{n}^{\prime}, x_{n}^{\prime \prime}\right)$

$$
=\left(\xi_{n}^{\prime} x_{1}^{\prime} x_{2}^{\prime} \ldots x_{n}^{\prime}, \xi_{n}^{\prime \prime} x_{1}^{\prime \prime} x_{2}^{\prime \prime} \ldots x_{n}^{\prime \prime}\right) \text {. }
$$

If $X^{\prime}$ is a character for $\xi^{\prime}$ and $\chi^{\prime \prime}$ is a character for $\xi^{\prime \prime}$ then X: $M^{\prime} \times M^{\prime \prime} \rightarrow \mathrm{C}$ with

$$
\operatorname{dom} \chi=\operatorname{dom} \chi^{\prime} \times \operatorname{dom} \chi^{\prime \prime}
$$

and

$$
\chi\left(m^{\prime}, m^{\prime \prime}\right)=\chi^{\prime}\left(m^{\prime}\right) \chi^{\prime \prime}\left(m^{\prime \prime}\right),
$$

for all $m^{\prime}$ in dom $\chi^{\prime}$ and all $m^{\prime \prime}$ in dom $\chi^{\prime \prime}$, is a character of $\xi$. 
However it is not immediately obvious that a general character of $\xi$ must be of the form (1.2) where $\chi^{\prime}$ and $\chi^{\prime \prime}$ are characters of $\xi^{\prime}$ and $\xi^{\prime \prime}$ respectively, and it is our purpose in this paper to show that this is so. However we require a preliminary result about identity elements for summary functions and this is established in the next section.

\section{Adjunction of an identity element}

Let $X$ be a non-empty set and let $\xi: X_{*} \rightarrow M$ be a universal $M$-valued summary function. We say that $\xi$ has an identity element $e$ when there is an element $e$ in $X$ such that, for any positive integer $n>1$ and any elements $x_{1}, \ldots, x_{k-1}, x_{k+1}, \ldots, x_{n}$ in $\mathrm{X}$,

$$
\xi_{n} x_{1} \cdots x_{k-1} e x_{k+1} \cdots x_{n}=\xi_{n-1} x_{1} \ldots x_{k-1} x_{k+1} \cdots x_{n} \text {. }
$$

In particular

$$
\xi_{n} e e \ldots e=\xi_{1} e
$$

When $\xi$ does have an identity $e$ one has $\chi\left(\xi_{1} e\right)=1$ for any non-trivial character $X$ of $\xi$.

If $\xi$ does not have an identity element we can adjoin one to $x$ in the following way. Let $e$ be any symbol not representing an element of $X_{*}$ and write $y=X \cup e$. Similarly let $g$ be any symbol not representing an element of $M$ and write $L=M \cup g$. Define $\eta: V_{*} \rightarrow L$ by

(i) $\eta \mid x_{*}=\xi$,

(ii) $n_{n} e e \ldots e=n_{1} e=g, n \geq 1$,

(iii) for $n>1$ and any $\left(y_{1}, y_{2}, \ldots, y_{n}\right)$ in $y_{*}$ with exactly $k<n$ occurrences of $e$,

$$
n_{n} y_{1} y_{2} \cdots y_{n}=\xi_{n-k} x_{1} x_{2} \cdots x_{n-k}
$$

where $\left(x_{1}, x_{2}, \ldots, x_{n-k}\right)$ is the sequence obtained from $\left(y_{1}, y_{2}, \ldots, y_{n}\right)$ by deleting the $k$ occurrences of $e$.

It is easily verified that $\eta$ is a universal L-valued summary function with identity $e$. Moreover if $\alpha: L \rightarrow C$ is a non-trivial 
character of $\eta$ then $\alpha(g)=1$ and $x=\alpha \mid$ codom $\xi$ is a character of $\xi$. Conversely if $X$ is a character of $\xi$ and we define $\alpha:$ codomn $\rightarrow C$ by extension from $\alpha \mid \operatorname{codom} \xi=X$ and $\alpha(g)=1$ then $\alpha$ is a character of $\eta$. It follows that there is no loss of generality in supposing that a summary function does have an identity element. It should be noted, however, that an identity element for a summary function $\xi$ is not necessarily unique; if $e$ and $f$ are identities then we have $\xi_{1} e=\xi_{l} f$.

\section{The theorem}

We return now to the situation of Section 1 involving two summary functions $\xi^{\prime}$ and $\xi^{\prime \prime}$ and the derived summary function $\xi$ defined by equation (1.1). We will, however, suppose now that $\xi^{\prime}$ has an identity $e^{\prime}$ and that $\xi^{\prime \prime}$ has an identity $e^{\prime \prime}$. We start with the

LEMMA. Let $\mathrm{x}: \operatorname{codom} \xi \rightarrow \mathrm{C}$ be a character of $\xi$ and define $X^{\prime}: \operatorname{codom} \xi^{\prime} \rightarrow C$ by

$$
\forall m^{\prime} \in \operatorname{codom} \xi^{\prime}: \chi^{\prime}\left(m^{\prime}\right)=\chi\left(m^{\prime}, \xi_{I}^{\prime \prime} e^{\prime \prime}\right) .
$$

Then $X^{\prime}$ is a character of $\xi^{\prime}$. In like manner $\chi^{\prime \prime}:$ codom $\rightarrow C$ defined by

$$
\forall m^{\prime \prime} \in \operatorname{codom} \xi^{\prime \prime}: \chi^{\prime \prime}\left(m^{\prime \prime}\right)=\chi\left(\xi_{1}^{\prime} e^{\prime}, m^{\prime \prime}\right)
$$

is a character of $\xi^{\prime \prime}$.

Proof. For any $n \geq 1$ and any $x_{1}^{\prime}, x_{2}^{\prime}, \ldots, x_{n}^{\prime}$ in $X^{\prime}$ one has

$$
\begin{aligned}
\chi^{\prime}\left(\xi_{n}^{\prime} x_{1}^{\prime} x_{2}^{\prime} \ldots x_{n}^{\prime}\right) & =\chi\left(\xi_{n}^{\prime} x_{1}^{\prime} x_{2}^{\prime} \ldots x_{n}^{\prime}, \xi_{1}^{\prime \prime} e^{\prime \prime}\right) \\
& =\chi\left(\xi_{n}^{\prime} x_{1}^{\prime} x_{2}^{\prime} \ldots x_{n}^{\prime}, \xi_{n}^{\prime \prime} e^{\prime \prime} e^{\prime \prime} \ldots e^{\prime \prime}\right) \\
& =\chi\left(\xi_{n}\left(x_{1}^{\prime}, e^{\prime \prime}\right)\left(x_{2}^{\prime}, e^{\prime \prime}\right) \ldots\left(x_{n}^{\prime}, e^{\prime \prime}\right)\right) \\
& =\chi\left(\xi_{1}\left(x_{1}^{\prime}, e^{\prime \prime}\right)\right) \times\left(\xi_{1}\left(x_{2}^{\prime}, e^{\prime \prime}\right)\right) \ldots \times\left(\xi_{1}\left(x_{n}^{\prime}, e^{\prime \prime}\right)\right) \\
& =\chi\left(\xi_{1}^{\prime} x_{1}^{\prime}, \xi_{1}^{\prime \prime} e^{\prime \prime}\right) \times\left(\xi_{1}^{\prime} x_{2}^{\prime}, \xi_{1}^{\prime \prime} e^{\prime \prime}\right) \ldots \times\left(\xi_{1}^{\prime} x_{n}^{\prime}, \xi_{1}^{\prime \prime} e^{\prime \prime}\right) \\
& =\chi^{\prime}\left(\xi_{1}^{\prime} x_{1}^{\prime}\right) \chi^{\prime}\left(\xi_{1}^{\prime} x_{2}^{\prime}\right) \ldots \chi^{\prime}\left(\xi_{1}^{\prime} x_{n}^{\prime}\right) .
\end{aligned}
$$

This establishes that $X^{\prime}$ is a character of $\xi^{\prime}$ and the result for $X^{\prime \prime}$ is proved in the same way. 
We are now in a position to prove our main result, namely the

THEOREM. Let $x$ be a character of $\xi$. Then for any $\left(m^{\prime}, m^{\prime \prime}\right)$ in the codomain of $\xi$,

$$
\chi\left(m^{\prime}, m^{\prime \prime}\right)=\chi^{\prime}\left(m^{\prime}\right) \chi^{\prime \prime}\left(m^{\prime \prime}\right),
$$

where $x^{\prime}, \chi^{\prime \prime}$ are the characters of $\xi^{\prime}, \xi^{\prime \prime}$ respectively which are defined in the Zemma.

Proof. We start by establishing that

$$
\forall x^{\prime} \in X^{\prime} \quad \& \quad \forall x^{\prime \prime} \in X^{\prime \prime}: \chi\left(\xi_{1}\left(x^{\prime}, x^{\prime \prime}\right)\right)=\chi^{\prime}\left(\xi_{1}^{\prime} x^{\prime}\right) \chi^{\prime \prime}\left(\xi_{1}^{\prime \prime} x^{\prime \prime}\right) .
$$

To prove (3.2) we observe that

$$
\begin{aligned}
\chi\left(\xi_{1}\left(x^{\prime}, x^{\prime \prime}\right)\right) & =\chi\left(\xi_{1}^{\prime} x^{\prime}, \xi_{1}^{\prime \prime} x^{\prime \prime}\right) \\
& =\chi\left(\xi_{2}^{\prime} x^{\prime} e^{\prime}, \xi_{2}^{\prime \prime} e^{\prime \prime} x^{\prime \prime}\right) \\
& =\chi\left(\xi_{2}\left(x^{\prime}, e^{\prime \prime}\right)\left(e^{\prime}, x^{\prime \prime}\right)\right) \\
& =\chi\left(\xi_{1}\left(x^{\prime}, e^{\prime \prime}\right)\right) \chi\left(\xi_{1}\left(e^{\prime}, x^{\prime \prime}\right)\right) \\
& =\chi\left(\xi_{1}^{\prime} x^{\prime}, \xi_{1}^{\prime \prime} e^{\prime \prime}\right) \times\left(\xi_{1}^{\prime} e^{\prime}, \xi_{1}^{\prime \prime} x^{\prime \prime}\right) \\
& =\chi^{\prime}\left(\xi_{1}^{\prime} x^{\prime}\right) \chi^{\prime \prime}\left(\xi_{1}^{\prime \prime} x^{\prime \prime}\right) .
\end{aligned}
$$

Suppose now that $\left(m^{\prime}, m^{\prime \prime}\right)$ is in the codomain of $\xi$; then there is $n \geq 1$ and there are elements $x_{1}^{\prime}, x_{2}^{\prime}, \ldots, x_{n}^{\prime}$ in $X^{\prime}$ and elements $x_{1}^{\prime \prime}, x_{2}^{\prime \prime}, \ldots, x_{n}^{\prime \prime}$ in $X^{\prime \prime}$ such that

$$
m^{\prime}=\xi_{n}^{\prime} x_{1}^{\prime} x_{2}^{\prime} \ldots x_{n}^{\prime} \& m^{\prime \prime}=\xi_{n}^{\prime \prime} x_{1}^{\prime \prime} x_{2}^{\prime \prime} \ldots x_{n}^{\prime \prime} .
$$

To establish (3.1) we make use of (3.2) in observing that

$$
\begin{aligned}
\chi\left(m^{\prime}, m^{\prime \prime}\right) & =\chi\left(\xi_{n}^{\prime} x_{1}^{\prime} x_{2}^{\prime} \ldots x_{n}^{\prime}, \xi_{n}^{\prime \prime} x_{1}^{\prime \prime} x_{2}^{\prime \prime} \ldots x_{n}^{\prime \prime}\right) \\
& =\chi\left(\xi_{n}\left(x_{1}^{\prime}, x_{1}^{\prime \prime}\right)\left(x_{2}^{\prime}, x_{2}^{\prime \prime}\right) \ldots\left(x_{n}^{\prime}, x_{n}^{\prime \prime}\right)\right) \\
& =\prod_{k=1}^{n} x\left(\xi_{1}\left(x_{k}^{\prime}, x_{k}^{\prime \prime}\right)\right) \\
& =\left[\prod_{j=1}^{n} \chi^{\prime}\left(\xi_{1}^{\prime} x_{j}^{\prime}\right)\right]\left[\prod_{k=1}^{n} \chi^{\prime \prime}\left(\xi_{1}^{\prime \prime} x_{k}^{\prime \prime}\right)\right] \\
& =\chi^{\prime}\left(\xi_{n}^{\prime} x_{1}^{\prime} x_{2}^{\prime} \ldots x_{n}^{\prime}\right) \chi^{\prime \prime}\left(\xi_{n}^{\prime \prime} x_{1}^{\prime \prime} x_{2}^{\prime \prime} \ldots x_{n}^{\prime \prime}\right) \\
& =\chi^{\prime}\left(m^{\prime}\right) \chi^{\prime \prime}\left(m^{\prime \prime}\right) .
\end{aligned}
$$


This is the desired result.

Hote that the result just established can be easily extended to the

case of an arbitrary but finite number of component summary functions.

\section{Reference}

[1] Peter D. Finch, "Macroscopic descriptions of microscopic phenomena", Bulz. Austral. Math. Soc. 9 (1973), 391-410.

Department of Mathematics,

Monash University,

Clayton,

victoria. 